\title{
A Simulation based Performance Evaluation OF AODV, R-AODV AND PHR-AODV ROUTING PROTOCOLS FOR MOBILE AD HOC NETWORKS
}

\author{
Pravanjan Das ${ }^{1}$, Sumant Kumar Mohapatra ${ }^{2}$ and Biswa Ranjan Swain ${ }^{3}$ \\ ${ }^{1}$ Ericsson India Global Services Pvt. Ltd., Salt Lake, Kolkata, India \\ ${ }^{2}$ Trident Academy of Technology, Bhubaneswar, Odisha \\ ${ }^{3}$ Trident Academy of Technology, Bhubaneswar, Odisha \\ ABSTRACT
}

\begin{abstract}
Mobile Ad hoc Networks (MANETs) are characterized by open structure, lack of standard infrastructure and un-accessibility to the trusted servers. The performance of various MANET routing protocols is significantly affected due to frequently changing network topology, confined network resources and security of data packets. In this paper, a simulation based performance comparison of one of the most commonly used on-demand application oriented routing protocols, AODV (Ad hoc on-demand Distance Vector) and its optimized versions R-AODV (Reverse AODV) and PHR-AODV (Path hopping based Reverse $A O D V$ ) has been presented. Basically the paper evaluates these protocols based on a wide set of performance metrics by varying both the number of nodes and the nodes maximum speed. A NS-2 based simulation study shows that, as compared to AODV and PHR-AODV, R-AODV enhances the packet delivery fraction by $15-20 \%$ and reduces the latency approximately by $50 \%$. $R$-AODV requires lesser node energy for data transmission.
\end{abstract}

\section{KEYWORDS}

MANET, AODV, R-AODV, PHR-AODV, packet delivery fraction, latency.

\section{INTRODUCTION}

A group of mobile nodes made a MANET[1]. They form a network for information exchange. For this information exchange, they never use the central authority as well as existing fixed network infrastructure. This upcoming technology creates new research opportunities and dynamic challenges for different topology of the network, bandwidth limitation, node's battery capacity improvement and multi-hop communication.

The routes are updated at regular intervals with respect to their requirement in proactive routing but routes are determined only when there is a need to transmit a data packet in reactive routing. Single path routing protocols [1-2] and multipath routing protocols [3-5] are classified on the basis of number of routes computed between source and destination.

The area of discussion in this paper is based on AODV [2], a single path routing protocol and its multipath versions, R-AODV and PHR-AODV routing protocols. AODV is both on-demand and destination initiated, that means routes are established from destination only on demand [1]. But the problem in such kind of single path routing is the increased latency and packet loss due to 
dynamic nature of the routing environment. Unnecessary bandwidth consumption due to periodic beacons also affects the performance of AODV.

Besides, the performance of AODV is significantly affected due to the loss of single unicasted route reply (RREP) packets. The drastically altering environment prevents the RREP packet from getting delivered to the source node. As a result the source node starts rediscovery process, which in turn increases both consumed energy and communication delay. The Reverse-AODV (RAODV) [6], broadcasts the route reply packet throughout the network instead of unicasting it. This process generates multiple discovered partial or full disjoint paths at the source node. It also ensures both successful route discovery and data packet delivery reducing path fail correction messages.

Protecting network activity from intrusion of malicious nodes and enhancing the data security are the important issues of Mobile Ad hoc networks. Sometimes the performance of R-AODV routing protocol gets significantly affected due to the activity of these active malicious nodes. So PHR-AODV [11], builds multipath to destination and adaptively hops between the available paths for transmission of data packets. As a result load distribution arises and it ensures that the nodes do not get depleted of energy which in turn increases the network lifetime.

In this paper, a significant amount of network parameters and energy related parameters have been considered in order to compare the above mentioned routing protocols. The graphs obtained are based on multiple readings and later averaging for a single plot point. The simulation environment considered in this paper is highly dynamic (lesser pause time and higher nodes speed) and the simulation software used is network simulator (NS-2).

\section{OVERVIEW OF ROUTING PROTOCOLS}

This section briefly describes AODV, R-AODV and PHR-AODV routing protocols.

\subsection{AODV Routing Protocol}

The Ad hoc On-Demand Distance Vector (AODV) [1-2] is a destination initiated routing protocol that maintains the sequence number concept for loop free routing and initiates the route discovery process on demand, hence has the combined features of DSDV and DSR respectively. The entire working principle of AODV can be covered under two important phases: Route Discovery and Route Maintenance.

The node disseminates and avoids repeated processing of RREQ packets at nodes by matching the source IP address and RREQ ID pair of the packet with nodes stored information. A RREP packet is generated by a node if it is itself the destination node or it has an active route to the destination. The destination node unicasts the RREP packet back towards the source node along the reverse path.

Another route error known as RERR message initiates and defects a link break for for the next hop of an active route in it's routing table but it is not attempting for any other local repairing. As a decision the RERR message which is received looks for an another route from it's routing table.

\subsection{R-AODV Routing Protocol}

The Reverse AODV (R-AODV) [6], an optimized AODV routing protocol uses a reverse route discovery methodology in order to avoid RREP packet loss. R-AODV prevents a large number of 
retransmissions of RREQ packets which in turn reduces the congestion in the network and enhances the delivery ratio [7].

From the RREQ packet transmission point of view, both AODV and R-AODV play the same role. The RREQ packet format of R-AODV is same as that of AODV. When the destination node receives the first RREQ packet, a reverse route request (R-RREQ) packet is initiated and broadcasted to its neighbour nodes within its transmission range. The R-RREQ packet format is given in Table 1.

Table1. R-RREQ Message Format [6]

\begin{tabular}{|c|c|c|}
\hline Type & Reserved & Hop Count \\
\hline Broadcast ID \\
\hline Destination IP address \\
\hline Destination Sequence Number \\
\hline Source IP address \\
\hline Reply Time \\
\hline
\end{tabular}

The source node starts packet transmission after receiving the first R-RREQ message whereas the late arrived R-RREQ packets are saved for future use. A forward route entry is created if the node received R-RREQ packet is not a source node. Then the R-RREQ is broadcasted to it's neighbour nodes. After receiving the packet, it adds in a new path with a different hop or with the same next hop. This is totally depends upon a sequence number which is greater or less [7][9].

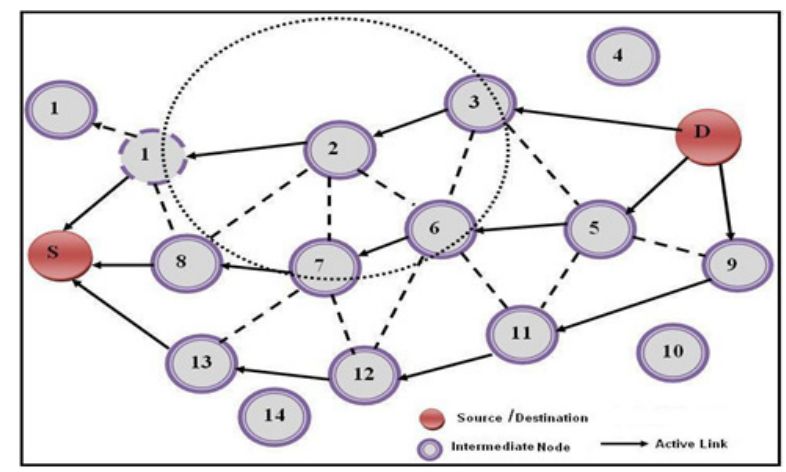

Figure1. R-RREQ from Destination to Source Node

Figure 1 shows the flooding of R-RREQ packet by the destination node in order to find the source node. So along with the primary path D-3-2-1-S, a number of paths might be built at the source node such as D-5-6-7-8-S, D-9-11-12-13-S.

\subsection{PHR-AODV Routing Protocol}

PHR-AODV [11] contains no permanent routes in nodes routing table. It is an extension of RAODV routing protocol which prevents loss of data packets by active malicious node and distributes load uniformly among the nodes.

The processing of RREQ and R-RREQ packets is same as that of R-AODV. The packet formats of R-AODV and PHR-AODV are same. When the source node receives R-RREQ packets from its neighbour nodes, it simply builds partial node-disjoint paths. After receiving all the node- 
disjoint paths within the timeout period, source node hops between different paths (based on the ascending order of their hop count values) while sending data packets to the destination node. During this communication of data packets, if a particular path fails then that path is eliminated from the list. The source node reinitiates the route discovery when no paths remain in the list. Figure 2 shows the node disjoint paths discovered between source and destination node.

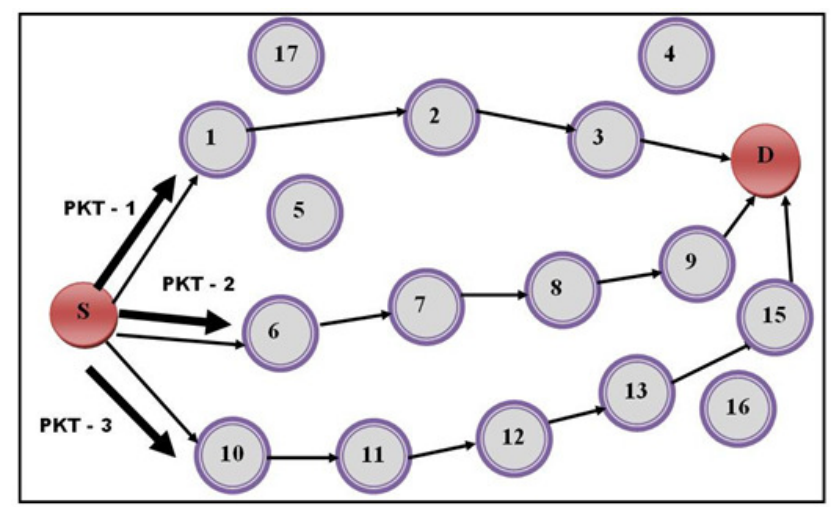

Figure2. Hopping of Paths at Source Node

PHR-AODV provides an effective and analytic method to estimate the security of the network [11]. The probability of active malicious node $\mathrm{Pm}$ is given as:

$$
\mathrm{Pm}=(\mathrm{Nrp} * \mathrm{Nm}) / \text { Ntotal }
$$

Where Nrp is the number of nodes in routing path, Ntotal is the number of all nodes in network, $\mathrm{Nm}$ is the Number of malicious nodes ( 2 malicious nodes are considered in this paper) and $\mathrm{Np}$ is the number of paths from a source to a destination. The malicious node intrusion rate is given by

$$
\mathrm{Pi}=\mathrm{Pm} / \mathrm{Np}
$$

\section{SIMULATION AND PERFORMANCE EVALUATION}

This section explained the simulation model and their performance metrics. This also describes the results which are analysed from the simulation.

\subsection{Simulation Model}

In this section, the network simulator-2 is used. It supports for simulating a multi-hop wireless adhoc environment completed with physical, data link and medium access control layer models. The table- 2 and 3 shows the simulation parameters and energy parameters respectively. 
Table 2. Energy Parameters [7][9]

\begin{tabular}{|c|c|}
\hline Parameters & Values \\
\hline Routing Protocol & AODV,R-AODV,PHR-AODV \\
\hline Number of Nodes & $20,30,40,50,60,70$ \\
\hline Area of Terrain & $1500 \mathrm{~m} \mathrm{X} 1500 \mathrm{~m}$ \\
\hline Total time for simulation & $100.0 \mathrm{sec}$ \\
\hline Type of Traffic & CBR \\
\hline Size of Packet & 512 bytes \\
\hline Maximum speed of Nodes & $2,5,10,15,20 \mathrm{~m} / \mathrm{s}$ \\
\hline Bandwidth & $2.0 \mathrm{Mbps}$ \\
\hline Transmission Range & $250 \mathrm{~m}$ \\
\hline Transmission Rate & 4 Packets/sec \\
\hline No of flows & 10 \\
\hline Frequency Band & $2.4 \mathrm{GHz}$ \\
\hline Pause Time & $5 \mathrm{sec}$ \\
\hline Propagation type & Propagation/TwoRayGround \\
\hline Antenna & Antenna/OmniAntenna \\
\hline Queue Type & Queue/DropTail/PriQueue \\
\hline Queue Length & 50 \\
\hline MAC type & Mac/802_11b \\
\hline Mobility Model & Random Waypoint \\
\hline
\end{tabular}

Table 3:Simulation Parameters

\subsection{Performance Metrics}

\begin{tabular}{|c|c|}
\hline Parameters & Values \\
\hline Receiving Power & 1.0 watt \\
\hline Transmitting Power & 1.4 watt \\
\hline Idle Power & 0.83 watt \\
\hline Sleep Power & 0.13 watt \\
\hline Transition Power & $0.2 \mathrm{watt}$ \\
\hline Nodes' Initial Energy & 300 Joules \\
\hline
\end{tabular}

The following performance metrics [6-10] are considered for the simulation:

- Packet Delivery Fraction: It is the ratio of data packets received at the destination to those generated by the source.

- Average End-to-End Delay: It is the time interval between Transmitting packet and Receiving packet, which is a summation of isolation of data packets during route discovery, retransmission delays at the MAC and queuing at the interface queue.

- Routing Overhead: It is the sum of all control packets transmitted/received during route discovery and route maintenance.

- Average Energy Consumed: It is the mean value of energy consumed by a node during the whole simulation process.

- Intrusion Rate: It is the percentage of intrusion instances present in the test set. 


\subsection{Results and Discussions}

The packet delivery fraction and average end-to-end delay of R-AODV characterisation are shown in figure 3 and 4 . It is very obvious that above two factors improved as compared to other two protocols due to R-AODV utilises multiple recent routes at the source node which are fresh enough. Also the routing overhead of R-AODV is much larger than AODV which is clearly shown in figure 5 because R-AODV broadcasts route reply packets whereas it is unicasted in AODV.

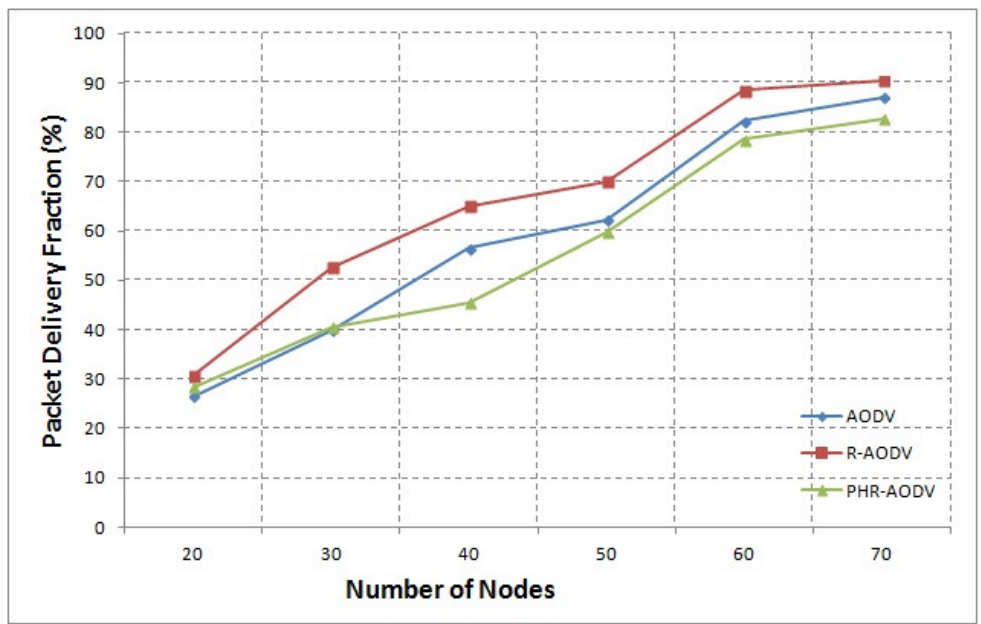

Figure 3. Packet Delivery Fraction varying Number of Nodes

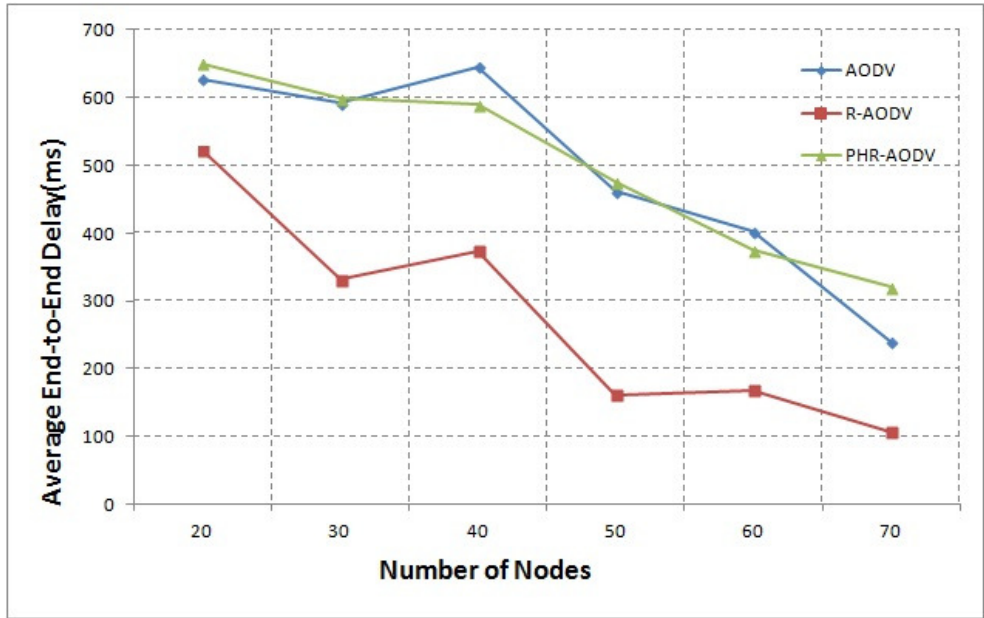

Figure 4. Average End-to-End Delay varying Number of Nodes 
International Journal of Wireless \& Mobile Networks (IJWMN) Vol. 6, No. 5, October 2014

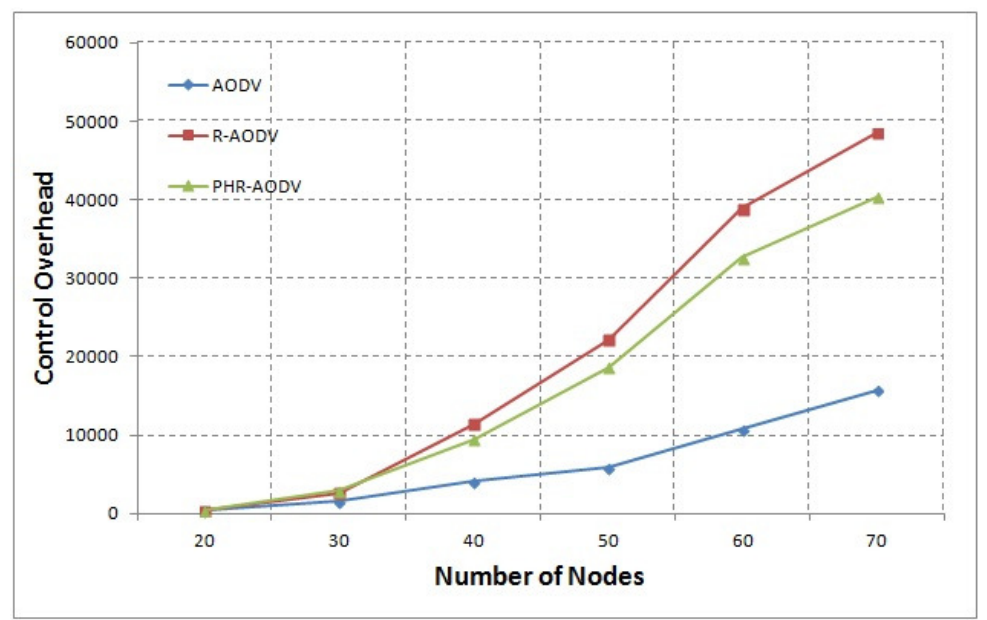

Figure 5. Control Overhead varying Number of Nodes

From Figure 6 and 7 it is clear that R-AODV completely outperforms AODV and PHR-AODV when performance is considered by varying Nodes Maximum Speed due to that R-AODV uses a number of multiple invented routes with high consistency to switch over the rapid topology changes due to higher nodes speed, which reduces the delay of communication which arises due to successful delivery of data packets.

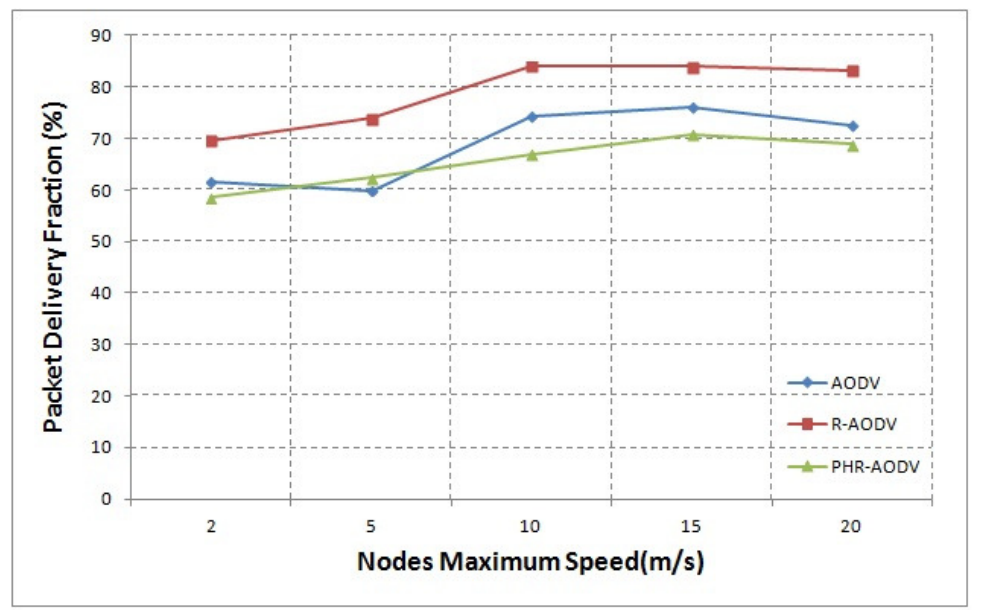

Figure 6. Packet Delivery Fraction vs. Nodes Max. Speed 
International Journal of Wireless \& Mobile Networks (IJWMN) Vol. 6, No. 5, October 2014

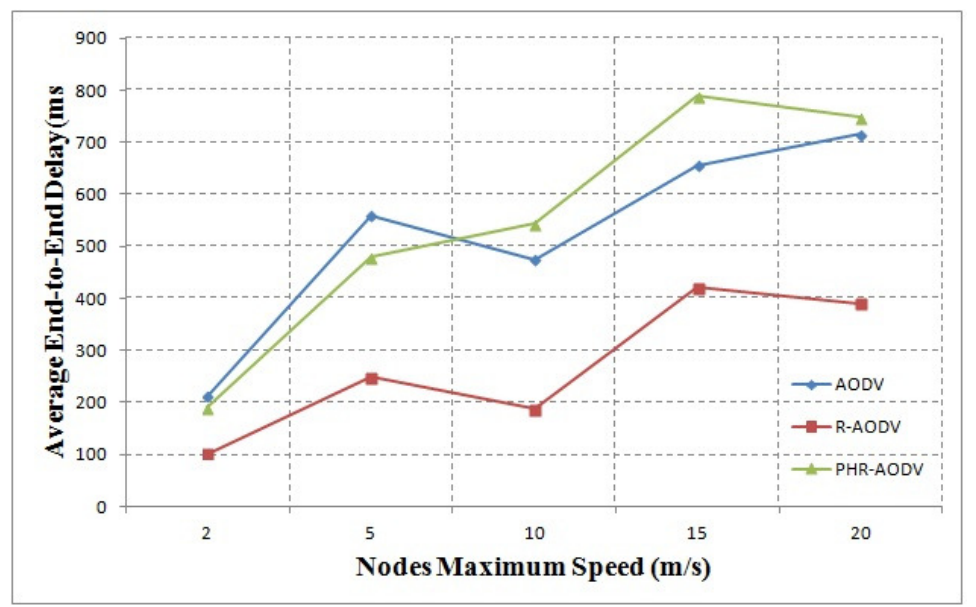

Figure 7. Average End-to-End Delay varying Nodes Max. Speed

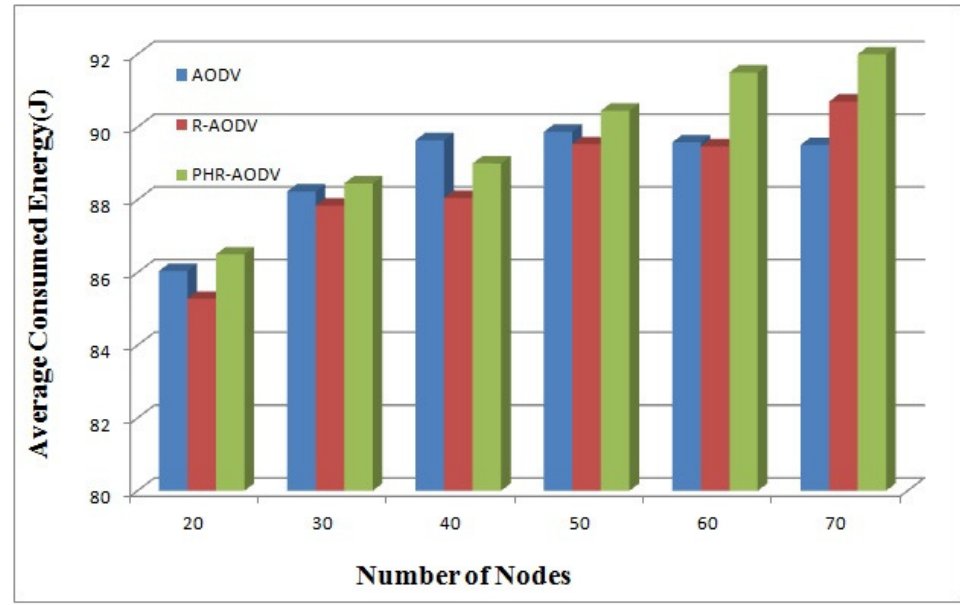

Figure 8. Average Energy Consumed varying Number of Nodes

The average consumed energy for R-AODV is lesser than AODV and PHR-AODV is clearly illustrated in figure(8). This will effective even if it has transmitted larger number of control packets than others. The reason is that the R-AODV uses fewer hops in the chosen paths to route the data packets[8]. As a result for a longer period R-AODV increases the survival of the nodes in the network. 


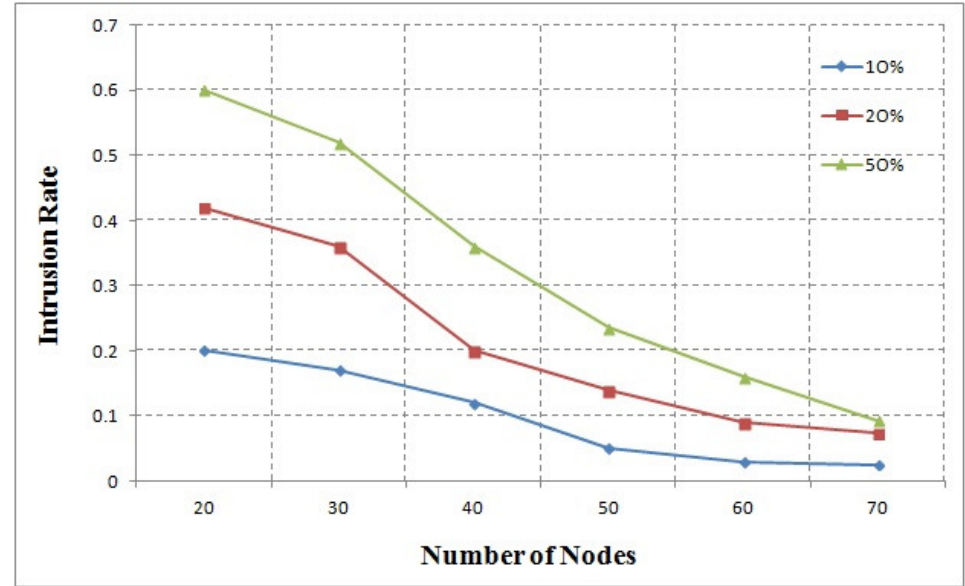

Figure 9. Intrusion Rate varying Number of Nodes

The two malicious nodes which are described in this paper, their intrusion rate decreases depending upon the number of nodes increases in case of PHR_AODV. Further the intrusion rate decreases as the probability of active malicious nodes decreases.

\section{Conclusions}

An extensive analytic model and a simulation study have been conducted to evaluate the performance of AODV, R-AODV and PHR-AODV using NS-2.The above simulation analysis raise a conclusion that the performance of R-AODV in terms average end-to-delay, average energy consumption and packet delivery fraction totally dominates both AODV and PHR-AODV .This is happened in high mobility scenarios at a cost of higher routing overhead. It can also be seen that in case of PHR-AODV, the intrusion rate of malicious nodes decreases with increase in number of nodes.

\section{ACKNOWLEDGEMENTS}

The authors would like to thank everyone, just everyone!

\section{REFERENCES}

[1] Elizabeth M. Royer and Chai-Keong Toh, "A Review of Current Routing Protocols for Ad Hoc Mobile Wireless Networks," IEEE Personal Communications, Vol. 6, No. 2, pp. 46-55, April 1999.

[2] C.E. Perkins and E. M. Royer, "Ad hoc on-demand distance vector routing" in Proc. WMCSA, New Orleans, LA, pp. 90-100, Feb. 1999.

[3] M. K. Marina and S. R. Das "On-Demand Multi Path Distance Vector Routing in Ad Hoc Networks” in Proc. ICNP 2001, pp. 14- 23, Nov. 2001.

[4] A. Nasipuri and S. R. Das, “On-Demand Multipath Routing for Mobile Ad Hoc Networks” Proc. ICCN 1999, pp. 64-70, Oct. 1999.

[5] Tarique, M., Tepe, E., Sasan Adibi, and Shervin Erfani, "Survey of multipath routing protocols for Mobile Ad-hoc networks", Journal of Network and Computer Applications, 32:1125-1143, 2009.

[6] Chonggun Kim, Elmurod Talipov and Byoungchul Ahn, "A Reverse AODV Routing Protocol in Ad Hoc Mobile Networks," LNCS 4097, pp. 522-531, 2006. 
International Journal of Wireless \& Mobile Networks (IJWMN) Vol. 6, No. 5, October 2014

[7] Pravanjan Das and Upena D Dalal, "A Comparative Analysis of AODV and R- AODV Routing Protocols in MANETS”, International Journal of Computer $\quad$ Applications $\quad 72(21): 1-5$, June 2013.

[8] Khafaei Taleb and Khafaie Behzad, "The Effect of Number of Hops per Path on Remind Energy in MANETs Routing Protocols," International Journal of Computer Applications vol. 43, no. 24, pp. 23 28, April 2012.

[9] Humaira Nishat, Vamsi Krishna K, D.Srinivasa Rao and Shakeel Ahmed, "Performance Evaluation of On-Demand Routing Protocols AODV and Modified AODV (R-AODV) in MANETS," International Journal of Distributed and Parallel Systems, vol. 2, no. 1, January 2011.

[10] Talipov, Elmurod, Donxue Jin, Jaeyoun Jung, Ilkhyu Ha, YoungJun Choi, and Chonggun Kim. "Path hopping based on reverse AODV for security." Management of Convergence Networks and Services, pp. 574-577. Springer Berlin Heidelberg, 2006.

[11] “The Network Simulator - NS-2”, available at http://www.isi.edu/nsnam/ns, 2004.

\section{Authors}

Sumant Ku Mohapatra was born in Bhubaneswar, Orissa, India. He received $t$ he B.Tech degree from ITER, Bhubaneswar, India in 2002 \& M.Tech(Electronics \& Instrumentation Engg) from GIET, BPUT, Odisha in 2011. Currently he is working in Trident Academy of Technology, B.P.U.T, Bhubaneswar, Odisha, India as an Assistant Professor. His research interest are in signal and image processing, optical fiber communication and wireless communication.

Pravanjan Das was born in the Cuttack district, Odisha, India . He received the B.Tech degree from TAT, Bhubaneswar, India in 2010 \& M.Tech(Communication Systems) from SVNIT,Surat,Gujrat in 2013. Currently he is working in Ericsson India Global Services Pvt. Ltd, Salt Lake, Kolkata, India as Researcher. His research interest are in signal processing, and wireless communication.

Biswa Ranjan Swain was born in the Cuttack district, Odisha, India. He received the B.Tech degree from SIET, Dhenkanal, India in 2006 \& M.Tech(Electronics \& Telecommunication Engg.) from IIT Kharagpur, Kharagpur, India. Currently he is working in Trident Academy of Technology, B.P.U.T, Bhubaneswar, Odisha, as an Assistant Professor. His research interest are in image processing, and wireless communication.
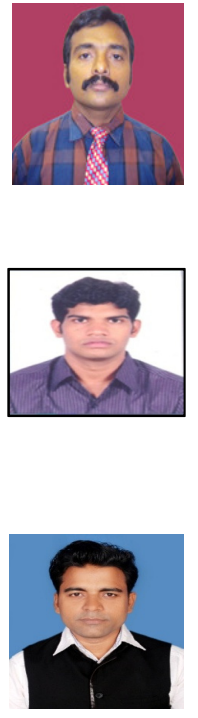